\title{
Suggestions for an interstellar cyclopropene search ${ }^{\star}$
}

\author{
A. K. Sharma ${ }^{\star \star}$ and S. Chandra ${ }^{\star \star \star}$ \\ School of Physical Sciences, Swami Ramanand Teerth Marathwada University, Nanded 431 606, India \\ Received 13 December 1999 / Accepted 25 May 2001

\begin{abstract}
Following tentative detection of cyclopropene $\left(\mathrm{C}_{3} \mathrm{H}_{4}\right)$ in Sgr B2 through its transition $3_{22}-2_{21}$, several attempts to confirm the presence of cyclopropene in astronomical objects (including Sgr B2 itself) have been made. We suggest that cyclopropene may be observed in astronomical objects through its transition $2_{20}-2_{21}$ at $3.67218 \mathrm{GHz}$, in absorption, even against the cosmic $2.7 \mathrm{~K}$ background, in a region having low density and low kinetic temperature.
\end{abstract}

Key words. ISM: molecules - molecular data

With the discovery of cyclopropenylidene $\left(\mathrm{C}_{3} \mathrm{H}_{2}\right)$ in a large number of astronomical objects (see, e.g., Madden et al. 1989), cyclopropene $\left(\mathrm{C}_{3} \mathrm{H}_{4}\right)$ has become a plausible candidate for detection in astronomical objects. A weak line at $106.86 \mathrm{GHz}$ in Sgr B2 observed by Thaddeus et al. (1985) coincided with one of the predicted strongest lines, the $3_{22}-2_{21}$ transition, of $\mathrm{C}_{3} \mathrm{H}_{4}$. Following this tentative detection of cyclopropene in Sgr B2, several attempts have been made to confirm its presence in astronomical objects (including Sgr B2 itself).

In order to provide rotational frequencies of cyclopropene throughout the radio spectrum to an accuracy sufficient for astronomical purposes, Vrtilek et al. (1987) reported the radio spectrum of cyclopropene. In Sgr B2, a search with the Bell Laboratories $7 \mathrm{~m}$ telescope for the $5_{15}-4_{14}$ and $5_{05}-4_{04}$ ortho-para line pair at $149.279 \mathrm{GHz}$ and $149.549 \mathrm{GHz}$, respectively, resulted in an upper limit of column density $5 \times 10^{14} \mathrm{~cm}^{-2}$, assuming a line width of $24 \mathrm{~km} \mathrm{~s}^{-1}$ and a rotational temperature of $11 \mathrm{~K}$ (Vrtilek et al. 1987). This upper limit was found to lie below the tentative detection (which implied the column density of $\mathrm{C}_{3} \mathrm{H}_{4}$ to be $1.3 \times 10^{15} \mathrm{~cm}^{-2}$ ), but is still somewhat above the measurement for $\mathrm{C}_{3} \mathrm{H}_{2}\left(6 \times 10^{13} \mathrm{~cm}^{-2}\right)$.

Send offprint requests to: S. Chandra, e-mail: chandrasuresh@123india.com

* Tables 1a and 1b are only available in electronic form at the CDS via anonymous ftp to

cdsarc.u-strasbg.fr (130.79.128.5) or via

http:// cdsweb.u-strasbg.fr/cgi-bin/qcat?J/A+A/376/333

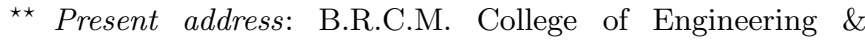
Technology, Bahal 127 028, India.

$\star \star \star$ Visiting Associate of the Inter-University Centre for Astronomy \& Astrophysics, Pune 411 007, India.
Cyclopropene is a cyclic, asymmetric top molecule with the electric dipole moment of $0.45 \mathrm{D}$ (Kasai et al. 1958) along the $a$-axis of inertia. In the present investigation, the NLTE occupation numbers of the $\mathrm{C}_{3} \mathrm{H}_{4}$ molecules are calculated in an on-the-spot approximation, by using the escape probability method (see, e.g., Rausch et al. 1996), where the external radiation field, impinging on the volume element emitting the line(s), is the cosmic $2.7 \mathrm{~K}$ background only.

The molecular data required as input for the present investigation are: (i) Einstein coefficients for various radiative transitions between the rotational energy levels accounted for, and (ii) the rate coefficients for collisional transitions between the levels due to collisions with $\mathrm{H}_{2}$ molecules. The details for calculation of Einstein A-coefficients for $a$-type rotational transitions in an asymmetric top molecule have been discussed by Chandra \& Rashmi (1998). These transitions are governed by the selection rules:

$$
\begin{array}{lll}
J: & \triangle J=0, \pm 1 & \\
k_{a}, k_{c}: & \text { odd, odd } \longleftrightarrow \text { odd, even } & \text { ortho-transitions } \\
& \text { even, even } \longleftrightarrow \text { even, odd } & \text { para-transitions. }
\end{array}
$$

The Einstein A-coefficients for the rotational transitions between the levels up to $70 \mathrm{~cm}^{-1}$ have been calculated by using the molecular and distortional constants derived by Vrtilek et al. (1987), and have been reported in Tables $1 \mathrm{a}$ and $\mathrm{b}$, for ortho- and para- $\mathrm{C}_{3} \mathrm{H}_{4}$, respectively, which are available in electronic form via anonymous ftp 130.79.128.5 at the CDS. (Though Vrtilek et al. 1987 reported line-intensities for a number of lines of $\mathrm{C}_{3} \mathrm{H}_{4}$, for a complete and consistent set of radiative transition probabilities, the Einstein A-coefficients are calculated in the present investigation.) 


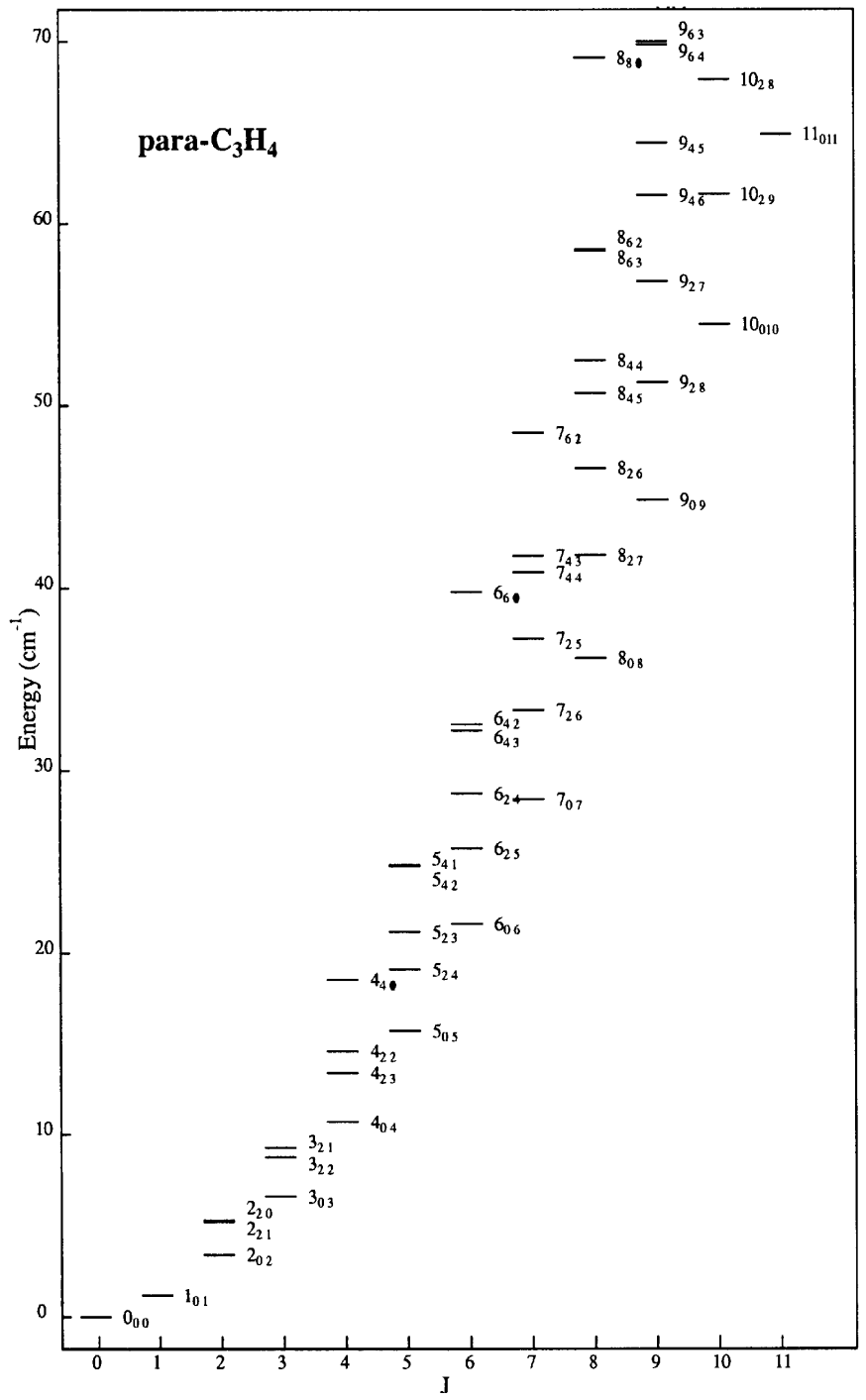

Fig. 1. Rotational energy levels in the ground vibrational state of para- $\mathrm{C}_{3} \mathrm{H}_{4}$, accounted for in the present investigation.

As of today, knowledge of the collisional transitions, particularly in asymmetric top molecules, is very poor. Furthermore, there are no data for the collisional rates for cyclopropene available in the literature. In the absence of any knowledge of collisional rates, we assumed that the collisional rate coefficient for a downward transition $J^{\prime} k_{a}^{\prime} k_{c}^{\prime} \rightarrow J k_{a} k_{c}$ at temperature $T(\mathrm{~K})$ is given by

$C\left(J^{\prime} k_{a}^{\prime} k_{c}^{\prime} \rightarrow J k_{a} k_{c}\right)=\left[1 \times 10^{-11} /\left(2 J^{\prime}+1\right)\right] \sqrt{T / 30}$.

The rate coefficient for the corresponding upward transition $J k_{a} k_{c} \rightarrow J^{\prime} k_{a}^{\prime} k_{c}^{\prime}$ has been calculated with the help of the detailed equilibrium equation.

In order to include a large number of astronomical objects where the molecule may be observed, numerical calculations are carried out for wide ranges of physical parameters. The molecular hydrogen density has been varied over the range from $10^{3} \mathrm{~cm}^{-3}$ to $10^{6} \mathrm{~cm}^{-3}$, and the calculations are performed for the kinetic temperatures of 10 , 20 and $30 \mathrm{~K}$. The transition $2_{20}-2_{21}$ at $3.67218 \mathrm{GHz}$, proposed for detection in astronomical objects, belongs
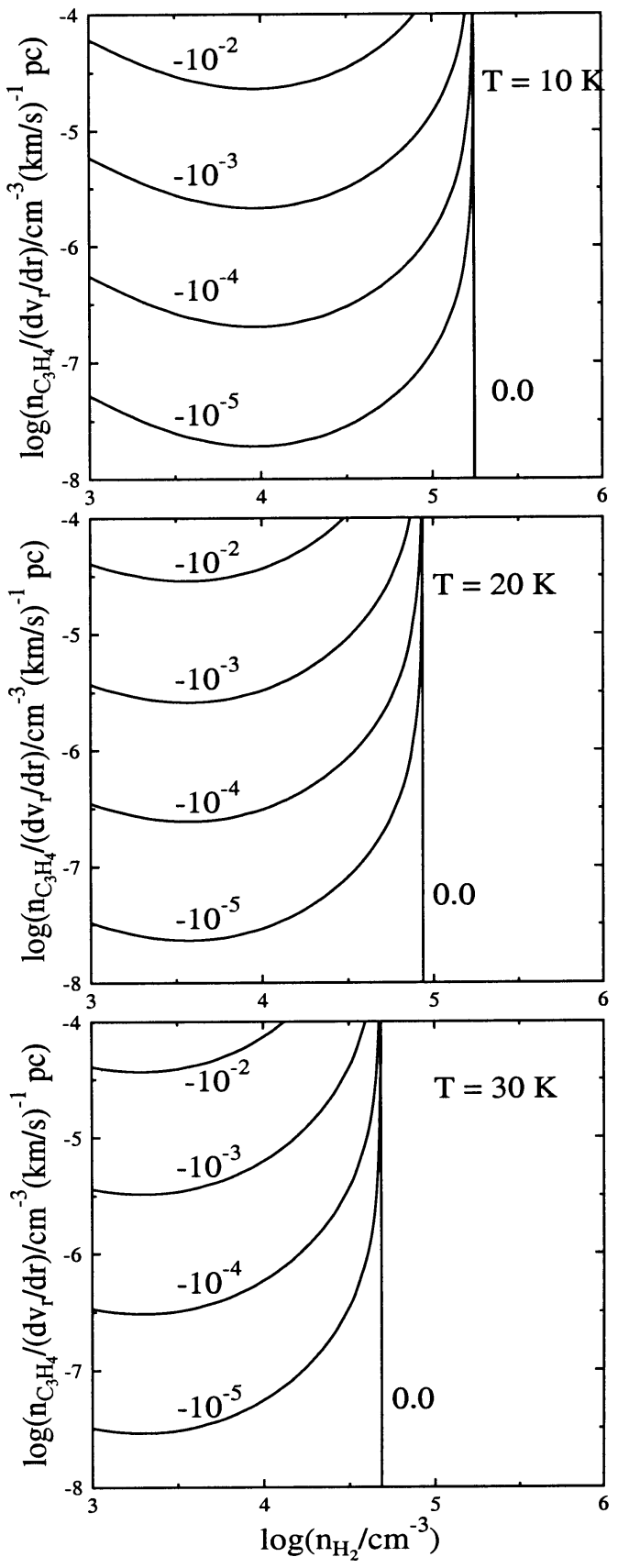

Fig. 2. The iso-lines for intensity against the cosmic $2.7 \mathrm{~K}$ background, in the unit of Planck's function at the kinetic temperature of $T(\mathrm{~K})$, i.e., $\left(I_{\nu}-I_{\nu, \mathrm{bg}}\right) / B_{\nu}(T)$, for the $2_{20}-2_{21}$ transition at $3.67218 \mathrm{GHz}$ of para- $\mathrm{C}_{3} \mathrm{H}_{4}$. Only negative values for the kinetic temperatures $T=10,20$, and $30 \mathrm{~K}$ are plotted. For large molecular hydrogen densities (where the iso-lines are not plotted), the value becomes positive.

to para- $\mathrm{C}_{3} \mathrm{H}_{4}$. For para- $\mathrm{C}_{3} \mathrm{H}_{4}$, we accounted for 52 rotational energy levels, shown in Fig. 1. These levels are connected through 217 radiative transitions for which the Einstein A-coefficients are given in Table 1b. In the calculations, the free parameters are hydrogen density $n_{\mathrm{H}_{2}}$, and $\gamma \equiv n_{\mathrm{C}_{3} \mathrm{H}_{4}} /\left(\mathrm{d} v_{\mathrm{r}} / \mathrm{d} r\right)$, where $n_{\mathrm{C}_{3} \mathrm{H}_{4}}$ is the density of $\mathrm{C}_{3} \mathrm{H}_{4}$, and $\left(\mathrm{d} v_{\mathrm{r}} / \mathrm{d} r\right)$ is the velocity gradient. The intensity, $I_{\nu}$, of a line generated in an interstellar cloud, with 
homogeneous excitation conditions, is given by

$I_{\nu}-I_{\nu, \mathrm{bg}}=\left(S_{\nu}-I_{\nu, \mathrm{bg}}\right)\left(1-\mathrm{e}^{-\tau_{\nu}}\right)$

where $I_{\nu, \mathrm{bg}}$ is the intensity of the continuum against which the line is observed, $\tau_{\nu}$ the optical depth of the line, and $S_{\nu}$ the source function, which is the Planck's function at the excitation temperature $T_{\mathrm{ex}}$, i.e., $S_{\nu}=B_{\nu}\left(T_{\mathrm{ex}}\right)$.

Figure 2 shows the iso-lines of intensity for the transition $2_{20}-2_{21}$, in the units of Planck's function $\left[\left(I_{\nu}-I_{\nu, \mathrm{bg}}\right) / B_{\nu}(T)\right]$, for the kinetic temperatures $T=10$, 20 , and $30 \mathrm{~K}$. In the figure, we have plotted only negative intensities; in the large density region (on the right-side of iso-lines), the intensity becomes positive. Thus, the absorption and emission nature of the line may play a significant role in providing information about the limiting value of the density in the region. For the low density region, the line $2_{20}-2_{21}$ shows absorption, even against the cosmic $2.7 \mathrm{~K}$ background, whereas in the large density region, the transition shows an emission against the cosmic $2.7 \mathrm{~K}$ background. (If there is a source in the background of the object, the line would show absorption against the background source.) The negative value of $\left(I_{\nu}-I_{\nu, \mathrm{bg}}\right) / B_{\nu}(T)$ increases with the decrease of the molecular hydrogen density. Further, it increases with the decrease of the kinetic temperature $T$. Thus, cyclopropene has a large probability of detection through its transition $2_{20}-2_{21}$ in cosmic objects having low density and low kinetic temperature.

Acknowledgements. We are grateful to Prof. Dr. W. H. Kegel of the University of Frankfurt/Main, Germany for his encouragement. Financial support from the D.S.T., New Delhi is gratefully acknowledged. Thanks are due to Mr. Harshal Hayatnagarkar for his valuable help.

\section{References}

Chandra, S., \& Rashmi 1998, A\&AS, 131, 137

Kasai, P. H., Myers, R. J., Eggers, D. F., \& Wiberg, K. B. 1958, J. Chem. Phys., 30, 512

Madden, S. C., Irvine, W. M., Matthews, H. E., Friberg, P., \& Swade, D. A. 1989, AJ, 97, 1403

Rausch, E., Kegel, W. H., Tsuji, T., \& Piehler, G. 1996, A\&A, 315,533

Thaddeus, P., Vrtilek, J. M., \& Gottlieb, C. A. 1985, ApJ, 299, L63

Vrtilek, J. M., Gottlieb, C. A., LePage, T. J., \& Thaddeus, P. 1987, ApJ, 316, 826 